\title{
Effektivität von Übungen im Zirkeltraining auf Gehgeschwindigkeit, Gleichgewicht und funktionelle Mobilität bei Schlaganfallpatienten: Eine Metaanalyse
}

Bonini-Rocha AC, de Andrade ALS, Moraes AM, Gomide Matheus LB et al. Effectiveness of Circuit-Based Exercises on Gait Speed, Balance, and Functional Mobility in People Affected by Stroke: A Meta-Analysis. PM R. 2017; DOI: https://doi.org/10.1016/j. pmrj.2017.09.014

Abstract aus dem Englischen übersetzt und zur Verständlichkeit leicht modifiziert von T. Neuendorf

\section{Hintergrund}

Es existieren mehrere Verfahren, die zur Rehabilitation von neurologisch betroffenen Patienten nach einem Schlaganfall in Frage kommen. Die Effektivität von Übungen in der Organisationsform des Kreisbzw. Zirkeltrainings, z.B. in Form eines übergreifenden Programms zur Verbesserung der Kraft, Ausdauer, Gleichgewichtsfähigkeit oder Alltagsfunktion, wurde bisher noch nicht dokumentiert.

\section{Ziel}

Die Untersuchung der Effektivität von Übungen im Zirkeltraining im Rahmen der Schlaganfallrehabilitation.

\section{Methoden}

Es wurde eine Literaturrecherche bei PubMed, Embase, Cochrane Libary und der Physiotherapy Evidence Database durchgeführt, um kontrollierte klinische Studien, unabhängig des Veröffentlichungszeitpunkts oder der Sprache, zu ermitteln. Die gesamte mittlere Differenz wurde für alle Therapieergebnisse mit einem Konfidenzintervall von $95 \%$ berechnet. 2 unabhängige Gutachter beurteilten das Risiko der Befangenheit und eventuellen Verzerrung.

\section{Ergebnisse}

11 Studien erfüllten die Einschlusskriterien, 8 davon präsentierten geeignete Daten, um eine Metaanalyse durchzuführen. Die quantitative Analyse zeigte, dass ein Zirkeltraining in Bezug auf die Gehgeschwindigkeit effektiver war als konventionelle Trainingsformen (mittlere Differenz von $0,11 \mathrm{~m} / \mathrm{s}$ ). Das Zirkeltraining war zudem nicht signifikant effektiver, um das Gleichgewicht oder die funktionelle Mobilität zu verbessern.

\section{Schlussfolgerung}

Die Ergebnisse demonstrieren, dass Zirkeltraining im Vergleich zu konventionellen Interventionen bessere Effekte für die Gehfähigkeit nach sich ziehen. Der Effekt auf die Gleichgewichtsfähigkeit und die funktionelle Mobilität fallen nicht besser aus als bei gewöhnlichen Trainingsformen.

\section{Evidenzlevel}

Muss noch bestimmt werden.

\section{Kommentar zur Studie}

Das Zirkeltraining beschreibt eine bekannte Organisationsform des Trainings, bei dem mehrere Stationen nacheinander durchlaufen werden. Eine günstige Beeinflussung relevanter Parameter hängt insbesondere von der Trainingsgestaltung sowie der Übungsauswahl ab. Variablen bei der Gestaltung eines Zirkeltrainings sind die Übungsdauer an einer Station sowie die Pausenzeiten dazwischen. Außerdem kann zwischen wenig bis hochintensiven Übungen variiert werden. Prinzipiell können in den Studien der hier aufgeführten Übersichtsarbeit benannte Elemente der Übungsinhalte aus dem Zirkeltraining auch im Rahmen der konventionellen Therapie integriert werden.
Auffällig sind die aufgeführten Trainingsinhalte im Rahmen der vorliegenden Metaanalyse, die sich nicht nur in der Organisation des Trainings (Kreis vs. konventionell), sondern auch in den Inhalten wesentlich unterscheiden. Eine Verbesserung der Ausdauerleistungsfähigkeit wird natürlich eher beim Training auf dem Laufband (Inhalt aus dem Zirkeltraining) erreicht als beim Üben mit Spielkarten (Inhalt aus dem konventionellen Training). Demnach sind die Übungsinhalte aus dem konventionellen Training zum Teil ungeeignet, um die hier bekannten Zielparameter Kraft, Ausdauer und Gleichgewicht positiv zu beeinflussen. Bei anderen bekannten Übungsinhalten aus der konventionellen Therapie hat sich eventuell bereits ein Gewöhnungseffekt eingestellt, weshalb die Patienten von Trainingsreiz nicht ähnlich profitieren wie von neuen Inhalten im Zirkeltraining. Die Trainingsinhalte beim Zirkeltraining aus den klinischen Studien erscheinen vielseitiger und anspruchsvoller als die konventionellen Übungsformen.

Eine Veränderung der Gehfähigkeit im Bereich von 0,1-0,2 m/s wird im Rahmen der Metaanalyse mit „minimaler klinischer Relevanz" eingestuft. Dementsprechend ist auch die dokumentierte mittlere Differenz von $0,11 \mathrm{~m} / \mathrm{s}$ einzuordnen. Die ausgeführten Studien unterscheiden sich stark hinsichtlich der Stichprobengröße ( $n=6$ bis $n=125$ für die Interventionsgruppe) und weisen teilweise eine große Streuung der Daten auf. Im nächsten Schritt sollte die Effektivität des Zirkeltrainings systematisch in Abhängigkeit des Krankheitsstadiums (akut, subakut, chronisch) bei hinreichend großen Stichproben untersucht werden. Außerdem könnte in weiteren Studien ermittelt werden, welche Parameter sich durch ein Zirkeltraining bei Schlaganfallpatienten am ehesten positiv beeinflussen lassen, um 
Therapieinhalte entsprechend zu planen und umzusetzen. Die vorliegende Metaanalyse präsentiert bisher vorliegende Daten aus den Jahren 2000-2012. Dabei wurden die Assessments „Berg Balance Scale“ (Gleichgewichtsfähigkeit), Timed Up\&Go Test (funktionelle Mobilität) sowie die Messung der Ganggeschwindigkeit durchgeführt. Nichtsdestotrotz ist ein Training im Kreisbetrieb, egal mit welchen spezifischen Übungen, im Rahmen der Schlaganfallrehabilitation denkbar. Besonders für ähnlich schwer betroffene Patienten mit vergleichbarem Ausprägungsgrad der Symptomatik und dementsprechendem Therapiebedarf könnte dies eine Möglichkeit zum Training in einer Kleingruppe darstellen. Psychosoziale Aspekte im gemeinsamen Training können eine wertvolle Ergänzung zur Individualtherapie mit einem Therapeuten pro Patient darstellen. Ein gegenseitiger Austausch und Kommunikation sowie die Motivationsförderung durch Spaß und Ansporn bei den Übungen könnte eine Abwechslung zum normalen Therapiealltag bei chronischen Schlaganfallpatienten darstellen. Auch in der Einzeltherapie kann der Therapeut verschiedene Stationen aufbauen, die dann im Kreis durchlaufen werden. Durch diese gezielte Vorbereitung der Therapiesitzung könnte die verfügbare Therapiezeit effektiver genutzt werden und sich die aktive Übungszeit erhöhen. Wie die Autoren andeuten könnten auch neue Therapieinhalte (z.B. im
Kontext der virtuellen Realität) einen entscheidenden Benefit auf genannte Parameter bewirken - eine Eignung im Rahmen eines Zirkeltrainings wäre diesbezüglich zu prüfen.

\section{Autorinnen/Autoren}

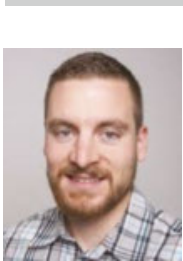

\section{Tilo Neuendorf}

Technische Universität Chemnitz Institut für Angewandte Bewegungswissenschaften, Professur Sportmedizin/biologie Thüringer Weg 11 09126 Chemnitz

E-Mail: tilo.neuendorf@hsw.tu-chemnitz.de 